Recepción: 20 / 04 / 2017

Aceptación: 20 / 05 / 2017

Publicación: 15 / 09 / 2017

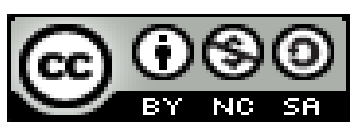

Ciencias de la salud

Artículo de revisión

\title{
Gestión del cuidado enfermero en el apego precoz y lactancia materna en el embarazo adolescente
}
Management of nursing care in early attachment and breastfeeding in adolescent pregnancy

\section{Gestión del cuidado enfermero en el apego precoz y lactancia materna en el embarazo adolescente}

Kristy G. Franco-Poveda ${ }^{\mathrm{I}}$ kristy.franco@cu.ucsg.edu.ec

Fanny de Lourdes Ronquillo-Moran II fanny.ronquillo@cu.ucsg.edu.ec

Martha L. Holguin-Jimenez III martha.holguin01@cu.ucsg.edu.ec

Lorena R. Gaona-Quezada ${ }^{\text {IV }}$ lorena.gaona@cu.ucsg.edu.ec

Correspondencia: kristy.franco@cu.ucsg.edu.ec

I. Magister en Gerencia y Administración en Salud; Diploma Superior en Gestión de Seguridad y Salud en el Trabajo; Licenciada en Enfermería; Universidad Católica Santiago de Guayaquil, Guayaquil, Ecuador.

II. Magister en Gerencia en Salud para el Desarrollo Local; Especialista en Gerencia y Planificación Estratégica de Salud; Diploma Superior de Cuarto Nivel en Desarrollo Local y Salud; Licenciada en Enfermería; Enfermera; Universidad Católica Santiago de Guayaquil, Guayaquil, Ecuador.

III. Magister en Gerencia en Salud para el Desarrollo Local; Licenciada en Enfermería; Universidad Católica Santiago de Guayaquil, Guayaquil, Ecuador.

IV. Magister en Docencia y Gerencia en Educación Superior; Especialista en Gerencia de Servicio de Salud; Magister en Epidemiologia y Enfermedades Tropicales; Licenciada en Enfermería; Universidad Católica Santiago de Guayaquil, Guayaquil, Ecuador. 
Gestión del cuidado enfermero en el apego precoz y lactancia materna en el embarazo adolescente

\title{
Resumen
}

Actualmente la mayoría de las madres conocen la importancia de la lactancia materna tanto para la madre como para el hijo desde el punto de vista nutritivo y de desarrollo así como afectivo y de prevención de patologías; pero algunas madres desconocen que una técnica de lactancia inadecuada puede llegar a ocasionar problemas tempranos en las mamas y con ello, en numerosas ocasiones, el abandono temprano del amamantamiento.

Palabras claves: Apego precoz; lactancia materna; embarazo adolescente.

\begin{abstract}
Most mothers are now aware of the importance of breastfeeding both for the mother and for the child from the nutritional and developmental point of view as well as affective and pathological prevention; but some mothers are unaware that an inadequate breastfeeding technique can lead to early problems in the breasts and with this, in many cases, the early abandonment ofbreastfeeding.
\end{abstract}

Key words: Early attachment; Breastfeeding; teenage pregnancy. 
Kristy G. Franco-Poveda; Fanny de Lourdes Ronquillo-Moran; Martha L. Holguin-Jimenez; Lorena R. Gaona-Quezada

\section{Introducción.}

El apego precoz desde los primero minutos de vida del recién nacido, inciden de manera positiva en el desarrollo físico, emocional e intelectual de los recién nacidos, este método tiene trascendencia mundial y nacional dentro de los protocolos de atención de enfermería, debido a que, el buen apego favorece los lazos afectivos entre el binomio madre hijo que se relaciona con la duración y calidad de la lactancia natural. (1)

Aunque los sentimientos de amor de la madre hacia su hijo recién nacido no son instantáneos, la primera hora parece tener una especial importancia en el establecimiento del vínculo afectivo. Es importante que durante esas primeras horas permanezcan juntos, a ser posible, en contacto piel con piel, así la madre se vincula intensamente, de forma totalmente inconsciente, con su hijo. (2)

La lactancia materna exclusiva es de vital importancia para la recuperación y mejoramiento del sistema reproductor femenino, debido a que la producción de la misma favorece la involución uterina por medio de la oxitocina natural, recuperación de peso, osteoporosis, cáncer de mama y ovarios. Además, disminuyen en el recién nacido patologías respiratorias como asma bronquial, atópicas, gastrointestinales, linfáticas, metabólicas, e inmunitarias; en comparación con los niños que no recibieron este privilegio. Además a largo plazo continúan en buena condición de salud y menor incidencia de enfermedades cardiovasculares, cerebrovasculares, autoinmunes y canceres. (3)

Las adolescentes menores de 16 años corren un riesgo de defunción materna cuatro veces más alto que las mujeres de 20 a 30 años, y la tasa de mortalidad de sus neonatos es aproximadamente un 50\% superior, según el consultor en salud de los adolescentes James E Rosen, de la OMS. Los expertos en salud convienen en que las adolescentes embarazadas requieren 
Gestión del cuidado enfermero en el apego precoz y lactancia materna en el embarazo adolescente

atención física y psicológica especial durante el embarazo, el parto y el puerperio para preservar su propia salud y la de sus bebés. (4)

En el Ecuador siguiendo la misma temática, se busca incrementar la lactancia materna exclusiva en los seis primeros meses de vida como una medida para contrarrestar los problemas de las madres especialmente adolescentes y evitar los riesgos de mortalidad materno neonatal. Por este motivo, se incrementó la lactancia materna exclusiva en los seis primeros meses de vida al $43 \%$ en el 2012 y es una de las 8 metas incluidas en el Plan Nacional del Buen Vivir 2013-2017.

El propósito de esta investigación es introducir al lector en actividades encaminadas a valorar la gestión del cuidado enfermero como herramienta exitosa para la atención oportuna de los cuidados enfermeros en el sistema de salud nacional y comunidad adolescente. Partiendo, de la necesidad de ejercer un liderazgo trasformador de recursos desperdiciados y construyendo estrategias que propaguen cuidados holísticos que integren el binomio madre hijo con la enfermera tratante.

El objetivo de la presentes investigacion es gestionar los principales problemas de la madre adolescente en el Apego precoz y Lactancia materna y los factores influyentes en este proceso para brindar cuidados de enfermería eficientes

\section{Metodología.}

Para lograr el objetivo de este estudio, se ha realizado una revisión narrativa de la literatura en las siguientes bases de datos: REDALYC, PUBMED y SCIELO. 
Kristy G. Franco-Poveda; Fanny de Lourdes Ronquillo-Moran; Martha L. Holguin-Jimenez; Lorena R. Gaona-Quezada

\section{Revision de Literatura.}

En Ecuador, según la Encuesta ENSANUT-ECU 2011-2013, sólo el 52\% de niñas y niños de hasta un mes de vida se alimentan exclusivamente con leche materna; y con el paso de los meses, el porcentaje tiende a disminuir notablemente. Muchos son los factores que inciden para que las madres no amamanten a sus hijos, algunas evidencias dan cuenta que es por falta de conocimiento sobre los beneficios de la lactancia materna, la falta de apoyo del personal de salud. (5)

UNICEF, conjuntamente con la OMS y el Ministerio de la Salud Pública, alerta y llama la atención sobre la importancia de intensificar las acciones para proteger, promover y apoyar la lactancia materna como una intervención clave para disminuir los índices de mortalidad y malnutrición infantil. (6)

La leche materna es un líquido molecularmente completo que representa la nutrición ideal del lactante, para su consumo exclusiva durante los primeros seis meses de vida, y complementaria a partir del séptimo mes hasta los 2 años de edad, de esta manera se asegura el desarrollo físico, emocional e intelectual de los niños y niñas. La leche materna proporciona al bebé la cantidad exacta de grasas, proteínas, vitaminas y azúcar. Contiene anticuerpos especiales que le brindan protección contra infecciones respiratorias, gripe, asma, infecciones de oído, diarreas y otras enfermedades. (7)

El organismo de la mujer está preparado para producir el alimento ideal del niño. La lactancia materna es la forma que la naturaleza tiene de proporcionar alimento al recién nacido, y con tal de que la mujer lo acepte y entienda lo que significa, es el comienzo ideal para una relación feliz y sana entre la madre y el hijo. (8) 
Gestión del cuidado enfermero en el apego precoz y lactancia materna en el embarazo adolescente

El apego precoz y lactancia materna debe iniciarse inmediatamente después del nacimiento, si es posible en el lugar donde se realizó el parto para establecer una relación estrecha entre la madre y el recién nacido. Otra de las razones es la importancia de brindar la primera leche, de color amarillo brillante conocida como calostro, la cual es vital por su función de protección al niño contra enfermedades, es de fácil digestión, tiene alto valor nutritivo y ayuda al niño a evacuar sus primeras heces. El principal estímulo que induce la producción de la leche es la succión del niño por lo tanto, cuantas más veces la madre le brinda el pecho y cuanto mejor se vacía éste, más leche se produce. La cantidad se ajusta a lo que el niño necesita y a las veces que vacía el pecho durante todo el día.

La Lactancia Materna no solo es asunto de la madre, sino también de los familiares: padre (quien debe apoyar con los deberes del hogar), además involucra al personal de salud quien es el encargado de orientar y brindar durante el período de embarazo toda la información necesaria sobre su importancia, así como también saber identificar los posibles problemas y sus soluciones. Con todo este apoyo se logrará una lactancia materna exclusiva y exitosa. (10)

En la actualidad, el embarazo en las adolescentes es un problema de salud pública y trae consigo un número elevado de complicaciones para la salud de la madre y de su hijo. Según un estudio realizado por James E. Rosen encargado de la investigación del Departamento de Reducción de los Riesgos del Embarazo de la Organización Mundial de la Salud (OMS), aproximadamente 16 millones de niñas entre los 15 y 19 años son madres adolescentes junto con ello la disminución de lactancia materna, la que no alcanza a llegar a los 6 meses de vida. (11)

El amamantamiento es una experiencia satisfactoria tanto para la madre como para el hijo por el contacto piel con piel que se produce entre ambos, la madre es extraordinariamente sensible 
Kristy G. Franco-Poveda; Fanny de Lourdes Ronquillo-Moran; Martha L. Holguin-Jimenez; Lorena R. Gaona-Quezada

durante la lactancia y su mayor preocupación gira en torno a la alimentación y cuidado de su hijo. Sin embargo, en las madres adolescentes, debido fundamentalmente a la etapa de la vida que están viviendo, al desconocimiento y falta de experiencia les provoca "angustia". La madre adolescente, es una mujer joven, necesita vivir esa etapa de su vida, ya que la maternidad temprana no la hace mujer adulta. Son tareas y desafíos que las jóvenes nunca han abordado y llevan a estas madres adolescentes a sentirse recargadas de trabajo y con una carga emocional muy fuerte, lo que constituye un gran riesgo para la Lactancia Materna. (12)

Las madres adolescentes pueden lograr una Lactancia Materna Exclusiva de al menos seis meses, si el equipo de salud apoya la labor educativa sobre este tema, desde la etapa preescolar en los establecimientos educacionales y junto con la comunidad realizan acciones de intervención y educación en los Centros de Salud, para estimular y convencer a nuestras adolescentes de las bondades y beneficios de la Lactancia Materna Exclusiva en los primeros seis meses de vida de los lactantes. (12)

El embarazo y la lactancia durante la adolescencia son factores que determinan el estado de nutrición de la mujer actual en la vida futura. Los cuidados propios del embarazo y la lactancia se suman a la especial atención que el adolescente requiere para su adecuado crecimiento y desarrollo. Es recomendable que las adolescentes embarazadas y lactantes tengan un seguimiento de su estado de nutrición desde el primer trimestre de gestación asegurando así el aporte energético y de nutrimentos específicos para ambos. (12) 
Gestión del cuidado enfermero en el apego precoz y lactancia materna en el embarazo adolescente

\section{Dificultades de lactancia materna}

Algunos problemas o inconvenientes en el proceso de establecimiento de la lactancia se deben en la mayoría de los casos sólo a la inexperiencia en ocasiones por cuestiones relacionadas a la succión el bebé o a la forma del pezón puede dificultarse. (13)

Según el doctor Juan Casado, médico jefe de pediatría de Hospital Niño Jesús, autor de El gran libro de pediatría nos que estos son los problemas principales de lactancia:

Dolor durante el amamantamiento: Durante los primeros días de la lactancia puede que nos resulte doloroso dar el pecho, pero esto es bastante normal. Es fácil sentir estas molestias ya que nuestra falta de práctica puede producirnos grietas o incluso coger alguna infección por hongos. Este dolor desaparecerá antes de la primera semana, aunque si persiste, quizás necesitemos de un tratamiento sencillo que no necesitará del abandono de la lactancia. (14)

Ingurgitación de las mamas: A partir del tercer día del parto, es frecuente sentir calor, dureza y congestión en los pechos, ya que la producción de leche aumenta rápidamente. A veces, esta congestión dificulta que el bebé se agarre eficazmente al pezón y debemos ayudarle apretando pezón con los dedos, intentando introducir toda la areola en su boca. Esta tensión mamaria es transitoria, irá desapareciendo a lo largo de los días. (15)

Pezones planos: El bebé puede tener dificultades en agarrar y mantener en su boca el pecho de su madre, cuando esta tiene los pezones planos o pequeños. Si este es el problema, debemos intentar apretar la areola mamaria para que salga el pezón o emplear pezoneras de caucho.

Pezones invertidos: Pueden verse un poco hundidos o directamente muy hundidos pero se retraen hacia atrás, en forma de ombligo, cuando son estimulados. 
Kristy G. Franco-Poveda; Fanny de Lourdes Ronquillo-Moran; Martha L. Holguin-Jimenez; Lorena R. Gaona-Quezada

Mastitis: La inflamación de la mama por la obstrucción o falta de drenaje de la mama tampoco es motivo suficiente renunciar a amamantar a tu hijo, aunque finalmente necesites tomar algún antiinflamatorio. Pese al dolor que puedas sentir en el pecho, sentirás alivio aplicando calor local y, especialmente, cuando tu bebé haya vaciado la leche de la mama inflamada. (16)

No tener suficiente leche: Una de las causas que más lleva al abandono prematuro de la lactancia es la creencia, generalmente errónea, de que no se tiene leche, que es de mala calidad. Curiosamente, la composición de la leche se mantiene incluso ante una malnutrición de la madre. La leche de la madre siempre es adecuada y satisface nutricionalmente al bebé. Hay que tener en cuenta que el bebé no tiene unos horarios estrictos de alimentación, ni que siempre que llora significa que tiene hambre. En una mujer sana, la producción de leche no siempre es la misma, puede disminuir por el nerviosismo, ansiedad. Si se da en el bebé un crecimiento deficiente, puede establecerse una lactancia mixta que permitirá igualmente que tú sigas amamantando a tu bebé y por tanto podrá seguir beneficiándose de los aportes tan extraordinarios de la leche materna. (17)

\section{Problemas psicosociales y patologias relacionadas al embarazo adolescente}

Los problemas psicosociales de mayor relevancia que afectan el apego precoz y lactancia materna exclusiva radican en el déficit de controles prenatales y nutricionales que se vinculan con aspectos culturales de la embarazada así como la trascendencia familiar del nuevo ser sin previo aviso, rechazando a la adolescente en etapa gestacional. Por ende, se asocian problemas de violencia intrafamiliar, migraciones, violaciones y drogadicción que con las políticas públicas nacionales connotativas al libertinaje dan pie a la falta de planificación familiar, drogadicción y hábitos culturas extranjeros que promueven el caos adolescente. Además de incluir los problemas de comunicación 
Gestión del cuidado enfermero en el apego precoz y lactancia materna en el embarazo adolescente

familiar y falta de tiempo para la atención oportuna de un adolescente con preguntas inconclusas e interrogantes que son tomadas en cuenta por amistades sin valores éticos morales definidos. (18)

Las patologías más frecuentes:

Anemia es una enfermedad por la que el organismo carece de la capacidad de producir la cantidad apropiada de hemoglobina (la proteína que transporta el oxígeno) o el número de glóbulos rojos. Los glóbulos rojos o hematíes son uno de los componentes del sistema sanguíneo y la hemoglobina es uno de sus principales componentes. (19)

Como los glóbulos rojos son los encargados de transportar el oxígeno de una célula a otra, el hecho de poseer una escasa cantidad significa que el organismo deberá trabajar con más intensidad para poder obtener y transportar el oxígeno necesario para los órganos y tejidos. (19)

En las mujeres embarazadas la anemia puede aparecer en distinto grado, especialmente a partir de la semana 20 de gestación.

\section{Infecciones de vías urinarias}

Información terapéutica del sistema nacional de salud (2005) no dice:

"Las infecciones del tracto urinario (ITU), son quizás las complicaciones médicas que más frecuentemente aparecen durante la gestación y que además pueden tener una repercusión importante tanto para la madre como para la evolución del embarazo. Las ITU pueden llegar a afectar al 5-10\% de todos los embarazos. Por ello se recomienda el cribado gestacional. Las modificaciones anatómicas y funcionales de la gestación incrementan el riesgo de ITU.’(pag.39) 
Kristy G. Franco-Poveda; Fanny de Lourdes Ronquillo-Moran; Martha L. Holguin-Jimenez; Lorena R. Gaona-Quezada

"Entre ellas destacan: hidronefrosis del embarazo, aumento del volumen vesical, disminución del tono vesical y ureteral, aumento de $\mathrm{pH}$ de la orina, éstasis urinario, aumento del reflujo vesicoureteral; glucosuria, menor «capacidad de defensa» del epitelio del aparato urinario bajo, incremento de la secreción urinaria de estrógenos y el ambiente hipertónico de la médula renal.”(pag. 39).

Las formas clínicas y frecuencia de presentación de ITU durante el embarazo son las siguientes:

- Bacteriuria asintomática: 2-11\%

- Cistitis: $1,5 \%$

- Pielonefritis aguda: 1-2\%

La diabetes gestacional es la alteración en el metabolismo de los hidratos de carbono que se detecta por primera vez durante el embarazo, esta traduce una insuficiente adaptación a la insulino resistencia que se produce en la gestante. (20)

Es la complicación más frecuente del embarazo y su frecuencia es variable según los distintos estudios, poblaciones y criterios diagnósticos utilizados.

Su importancia radica en que aumenta el riesgo de diversas complicaciones obstétricas como ser el sufrimiento fetal, macrosomía y problemas neonatales, entre otros. (20)

La preeclampsia es una afección propia de las mujeres embarazadas, que se caracteriza por la aparición de hipertensión arterial, y proteinuria, es decir, presencia de proteínas en la orina. Es un conjunto de signos y síntomas exclusivo de la gestación, aparece a partir de la semana 20 de 
Gestión del cuidado enfermero en el apego precoz y lactancia materna en el embarazo adolescente

embarazo, durante el parto o hasta las seis semanas postparto. Esta patología se caracteriza por una placenta anormal y disminución de la perfusión sistémica. (21)

La preeclampsia es más que una hipertensión arterial durante el embarazo que daña a los riñones provocando la pérdida de proteínas por la orina. El problema es que no solo se dañan los riñones durante el curso de la preeclampsia, sino que también hay afectación de otros órganos como el hígado, el cerebro y la propia sangre. Si no se controla la hipertensión comienzan a aparecer complicaciones que ponen en peligro la vida de la madre y del feto. (19)

\section{Virus de papiloma humano}

Según estudios realizados, en el Ecuador existen 726.010 mujeres en edad fértil según el censo poblacional del 2010 y se estima que por lo menos el $50 \%$ de los adultos sexualmente activos han presentado una infección por VPH genital en el transcurso de la vida. (22)

La infección por Virus Papiloma Humano (VPH) es una enfermedad de transmisión sexual (ETS), que afecta a un alto porcentaje de mujeres de todo el mundo, principalmente de países en desarrollo. La alta incidencia de infección por VPH está asociada al inicio precoz de la actividad sexual, mayor frecuencia de actividad sexual en la etapa de adolescentes y mayor número de parejas sexuales. (23)

La mayoría de las mujeres no saben de qué se trata la enfermedad. La ignorancia viene más de los sectores más pobres, donde tienen menos acceso a la poca información que se da sobre el VPH. (24)

La infección por el virus del papiloma humano (HPV) ha sido determinado como un factor etiológico para el desarrollo del cáncer de cuello uterino. Se han descrito más de 120 tipos de HPV y 
Kristy G. Franco-Poveda; Fanny de Lourdes Ronquillo-Moran; Martha L. Holguin-Jimenez; Lorena R. Gaona-Quezada aproximadamente una tercera parte de ellos son capaces de infectar el epitelio del tracto genital. Otros tumores relacionados con el HPV son del canal anal, vagina, vulva, pene y oral, de tal manera que se ha estimado que el HPV es responsable del 5,2\% de todos los cánceres en el mundo. (25)

Una persona puede infectarse varias veces por diferentes tipos de virus de papiloma a lo largo de su vida, e incluso al mismo tiempo. La infección es asintomática y en la mayoría de los casos, se cura de forma espontánea. No obstante, entre el $1 \%$ y el $2 \%$ de las infecciones persisten en el organismo y pueden dar lugar a desarrollar cáncer de cérvix. (7)

\section{Conclusion.}

La falta de gestión del cuidado efectiva radica en que la enfermería se centra en un trabajo multifuncional, con extenuantes horas laborables, mecánica compleja, protocolos con enfoque medico basados en el diagnóstico y tratamiento que desvanecen el arte del cuidado enfermero autónomo y responsable en la maternidad, desmejorado de manera radical los indicadores de morbimortalidad materno neonatal; enfatizando en este punto la importancia del rol de la enfermera educadora y promotora de la salud que proponga un plan de mejoramiento de la información brindada en sexualidad al adolescente desde los establecimientos educativos, sin incitar a la sexualidad precoz, más bien promoviendo estilos de vida que asocien los riesgos de embarazos no deseados a la búsqueda equivocada de influencias externas.

El principal problema de las madres adolescentes se debe al desconocimiento que tienen sobre Apego Precoz y Lactancia Materna. Los factores que inciden son la corta edad y el entorno emocional, agudizado en adolescentes de 13 a 16 años específicamente. Los del rango superior a 16 años, se enfocan mayormente en la situación económica y bajos niveles de educación. 
Gestión del cuidado enfermero en el apego precoz y lactancia materna en el embarazo adolescente

Los problemas psicosociales se caracterizan por la falta de comunicación familiar, la rotura de relaciones familiares, violencia intrafamiliar que llevan a la adolescente a buscar afecto equivocado con amistades nocivas que incitan muchas veces a hábitos tóxicos que conllevan a la gestación no planificada y muchas veces no aceptada en la comunidad.

Las adolescentes y sus parejas, mantienen relaciones sexuales no seguras por lo que mantiene embarazos no deseados, con riesgos para su salud del binomio madre hijo.

Los factores patológicos se relacionan con factores predisponentes como deficientes controles prenatales y mala alimentación. El bajo peso de la mujer embarazada contribuye a la restricción del crecimiento fetal, lo que aumenta el riesgo de mortalidad neonatal. Las patologías frecuentes en madres adolescentes, se relacionan con la presión arterial alta, infecciones de las vías urinarias, sin restar importancia a la anemia y las enfermedades de transmisión sexual como el virus del papiloma humano.

\section{Bibliografia.}

1. Morillo J, Montero L. Lactancia materna y relación materno filial en madres adolescentes. Enfermería Global. 2010 jul; 19(1): p. 1-9.

2. Cerda Muñoz L. Lactancia materna y gestión del cuidado. Revista Cubana de Enfermería. 2011 Dic; 27(4): p. 327-336.

3. Van Esterik P, Castro H, De Lima M. Lactancia Materna y Derechos. El derecho de todas las mujeres a amamantar. Revista Mujer Salud/Red de Salud de las Mujeres Latinoamericanas y del Caribe. 2008; 4(1): p. 55-56.

4. Rosabal Suárez L, Piedra Cosme B. Intervención de enfermería para la capacitación sobre lactancia materna. Revista Cubana de Enfermería. 2004 ene; 20(1).

5. Freire W, Ramírez M, Silva K, Romero N, Sáenz K. Encuesta Nacional de Salud y Nutricion ENSANUTECU 2011-2013 Quito: Ministerio de Salud Publica ; 2013.

6. UNICEF. Lactancia materna: un triunfo para toda la vida Quito: autor; 2014. 
Kristy G. Franco-Poveda; Fanny de Lourdes Ronquillo-Moran; Martha L. Holguin-Jimenez; Lorena R. Gaona-Quezada

7. OMS. La alimentación del lactante y del niño pequeño Capítulo Modelo para libros de texto dirigidos a estudiantes de medicina y otras ciencias de la salud Washington: Organización Panamericana de la Salud; 2010.

8. Estrada Rodríguez J, Amargós Ramírez J, Reyes Domínguez B, Guevara Basulto A. Intervención educativa sobre lactancia materna. Revista Archivo Médico de Camagüey. 2010 abr; 14(2).

9. González S, Cordi F, Robledo G, Valdespinos C, Varga V. La consejería en lactancia y contacto temprano en pareja madre-hijo están asociados a la lactancia materna exclusiva. Gac Med Mex. 2005; 2(1): p. 99-102.

10. Rosabal S, Piedra C. Intervención para la Capacitación sobre Lactancia. Rev Cubana Enfermería. 2004; 20(1).

11. OMS. Embarazo en adolescentes: un problema culturalmente complejo. Boletín de la Organización Mundial de la Salud. 2009; 87(1).

12. Reviriego. Problemas para amamantar al bebé. Guia Infantil. 2013.

13. Fernández Medina IM, González Fernández CT. Lactancia materna: prevención de problemas tempranos en las mamas mediante una técnica de amamantamiento eficaz. Enfermería Global. 2013 jul; 12(31): p. 443-451.

14. Hernández Aguilar M, Aguayo Maldonado J. La Lactancia materna. Cómo promover y apoyar la lactancia materna en la práctica pediátrica. Recomendaciones del Comité de Lactancia de la AEP. An Pediatr. 2005; 63(1): p. 340-356.

15. Britton C, McCormick F, Renfrew M, Wade A. Apoyo para la lactancia materna. The Cochrane Plus Database. 2008; 3(1).

16. Espínola-Docio B, Costa-Romero M, Díaz-Gómez M, Paricio-Talayero JM. Mastitis. Puesta al día. Archivos argentinos de pediatría. 2016; 114(6): p. 576-584.

17. OMS. Mastitis: causas y manejo Ginebra: WHO/FCH/ CAH; 2000.

18. Rodríguez Gázquez MdlÁ. Factores de riesgo para embarazo adolescente. Medicina UPB. 2008 jun; 27(1): p. 47-58.

19. Niswander K. Obstetricia práctica clinica Madrid: Reverté; 1987.

20. Almirón ME, Gamarra SC, González MS. Diabetes Gestacional. Revista de Posgrado de la VIa Cátedra de Medicina. 2005; 152(1): p. 23-27.

21. Macías Gaytán A. Preeclampsia. Tesis de Grado. Guayaquil: Universidad de Guayaquil, Facultad de Medicina; 2013.

22. Goyes Guerra MB, Jaramillo Parra AF, Moreira Macías JM. Prevalencia de infección por virus de papiloma humano de alto riesgo oncogenico (VPH-AR) en mujeres embarazadas que acuden al control por consulta externa en el hospital gineco obstetrico "Isidro Ayora" de la ciudad de Quito. Tesis. 
Gestión del cuidado enfermero en el apego precoz y lactancia materna en el embarazo adolescente

Guayaquil: Universidad de Guayaquil, Facultad de Ciencias Medicas ; 2015.

23. Concha P X, Urrutia S T, Riquelme H G. Creencias y virus papiloma humano. Revista chilena de obstetricia y ginecología. 2012; 77(2): p. 87 - 92.

24. Carrión Antonella FG. Comunicación para Mercadeo Social Virus del papiloma humano (VPH) en Ecuador y fundaciones relacionadas. Tesis (Licenciada en Comunicación Organizacional y Relaciones Públicas). Quito: Universidad San Francisco de Quito, Colegio de Comunicación y Artes Contemporáneas; 2012.

25. Zaldivar Lelo de Larrea. Cáncer cérvicouterino y virus del papiloma humano. Revista chilena de obstetricia y ginecología. 2012; 77(4): p. 315-321. 